\title{
La novela como experiencia de comunidad
}

\author{
The Novel as a Community Experience
}

\author{
Silvia BARDELÁs ÁlVAREZ \\ UNED \\ silviabardelas@gmail.com
}

Recibido: $15 / 07 / 2013$

Aceptado: 30/05/2014

\section{Resumen}

Este artículo propone una revisión de la novela como forma de arte autónoma transversal a la historia y adecuada a la sensibilidad estética actual.

Con referencias a la Filosofía de Jean-Luc Nancy, en la primera parte analiza cronológicamente algunas novelas percibiendo la presencia como una propiedad común. La segunda parte examina la crítica social como cualidad propia, crítica que parte de un impulso de búsqueda de las lógicas internas de la condición humana y que constituye en sí misma una experiencia de comunidad.

Palabras clave: novela, presencia, crítica social, experiencia, comunidad.

\begin{abstract}
This essay brings forward a review of the novel as an autonomous art form, transverse to history and suitable to current aesthetic sensibility. With references to the work of Jean Luc Nancy, the first part includes a historical analysis of the most representative novels revealing Presence as one of its fundamental characteristic. The second part examines social criticism as a trait of its own. This criticism reveals as a response to a distinguishing impulse: an open search of the internal logics of human condition, which is already in itself an experience of community.
\end{abstract}

Keywords: novel, presence, social critique, experience, community. 
La autonomía de la novela como forma artística se hace patente en el persistente debate sobre su muerte. Este debate, centrado en una supuesta incompetencia para reflejar la época actual, da pie a una reflexión que la ilumina justamente como una práctica artística muy adecuada para desvelar la sensibilidad contemporánea.

El objetivo de este artículo es mostrar cómo la novela no responde al gusto estético de una clase social en un determinado momento, no es una forma de narrar únicamente moderna, aunque coincida ese momento con su afianzamiento, sino más bien una disposición, una intención narrativa transversal a la historia y abierta a estilos propios.

Esa disposición que encontramos en las novelas más representativas desde la época griega hasta ahora tiene su correlato en el tipo de experiencia estética que suscita, una experiencia de comunidad alcanzada en la presencia al hilo de una crítica social.

Algunas de las ideas sobre las que Jean-Luc Nancy reflexiona, como la contraposición entre Gemeinschaft y Gesellschaft, la presencia en el arte, la distinción entre individuo y ser singular o la necesidad de la comunidad de revalorizarse continuamente en base a la falta de un valor primario iluminan esa forma de ser específica de la novela.

Estas son dos de las características fundamentales que la definen desde el punto de vista estético: la presencia, que le atribuye categoría de experiencia y un propósito de espejo crítico que concreta esa experiencia en experiencia de comunidad.

La literatura en el pensamiento de Jean-Luc Nancy no parece meramente un espacio de inspiración sino una experiencia estética que da cuenta de sus reflexiones sobre la comunidad.

\section{La presencia en la novela}

No es posible definir la novela como un género narrativo cerrado, como muy bien ha explicado Bajtín en su Teoría y estética de la novela ${ }^{1}$ Y tampoco es posible señalar un texto en la historia como primer texto novelístico. Su propio nombre no apunta más que a la novedad, no avanza nada con respecto a su forma, tampoco la palabra romance utilizada en otras lenguas hace referencia a nada más que a la lengua utilizada. A partir de su nombre, cualquier texto más o menos largo que narre una historia podría ser una novela. Sin embargo, existen rasgos comunes a textos narrativos representativos de todas las épocas desde la Grecia del siglo II hasta nuestros días que dan cuenta de la existencia de un impulso narrativo específico, una búsqueda de las lógicas internas de la condición humana alejado de cualquier ideología o concepción previa. Esta búsqueda libre se configura no en una mera representación de la re-

\footnotetext{
${ }^{1}$ Mijaíl M. Bakhtín, Teoría y estética de la novela, Madrid, Taurus, 1989.
} 
alidad, sino en una aparición de la misma, en la creación de una presencia que suscita una experiencia de comunidad que colma el impulso inicial.

Desde las llamadas novelas pastoriles y bizantinas de la época helenística hasta ahora, los textos que agrupamos como novelísticos no centran su interés en representar una idea, reproducir un mundo, relatar unos hechos, definir unos valores o tipos humanos, más bien buscan una experiencia de experiencias que desde una exploración crítica de su momento proporcionan una experiencia de comunidad. El lector no remata su lectura con un conocimiento conceptual, sino con un conocimiento intuitivo, una sensación que evidencia, pero en realidad, lo importante de ese conocimiento intuitivo es su carácter de experiencia y concretamente de experiencia de comunidad.

La presencia en cada novela se construye a través de una técnica original y adecuada para mostrar un mundo determinado. No hay definición, ni descripción en el sentido de enumerar las características de lo que ya vemos, hay intención de presencia, de poner en acto hechos reveladores. El lector se encuentra ante ellos conociendo lo propio, lo más íntimo, lo que no se puede describir ni explicar. Estar ante esa intimidad, precisamente es estar en presencia, en palabras de Jean-Luc Nancy: "La presencia no es una cualidad o una propiedad de la cosa. La presencia es el acto mediante el cual la cosa se pone por delante: prae-est"'2. Este carácter presencial, vocación novelística, viene dado por el impulso al que hacíamos alusión. La búsqueda sin ideología, la intención crítica hacia una forma de organización social que no permite el desarrollo de sus seres singulares lleva a mostrar la singularidad en la relación. De manera que la presencia en la novela es la presencia-con a la que alude Nancy: "Presencia-con: con en cuanto modo exclusivo del ser-presente, tal como el ser-presente, y el presente de ser, no coincide en sí- es decir, consigo- sino siempre que co-incide, que "cae con" otra presencia, la misma que a su vez obedece a la misma ley. El estarjunto-a-varios es la situación originaria: es incluso lo que define a una "situación" en general. Es así como un "con" originario o trascendental pide en adelante, con una urgencia sensible, liberarse y articularse por sí mismo"3. La novela muestra esa singularidad del ser que se da en su carácter finito precisamente al mostrar sus personajes en su con. De alguna manera el impulso colmado en la experiencia de la novela es ese con originario que pide liberarse como Nancy dice.

De manera que la presencia en la novela no es una cualidad buscada, no se puede decir que forme parte de una de las características que cierran un género, más bien surge naturalmente de la intención peculiar narrativa, del impulso que busca el estarcon y no lo común. Lo importante de los personajes novelísticos es su ser en relación,

${ }^{2}$ J. L. Nancy, La partición de las artes, Valencia, Pre-textos, 2013, p. 217.

${ }^{3}$ J. L. Nancy, La comunidad inoperante, Santiago de Chile, Escuela de Filosofía Universidad ARCIS, 2000, p. 126. 
el estar con otros que trasciende su individualidad permitiendo aflorar su singularidad $^{4}$. ¿Cómo podríamos ver la singularidad de Don Quijote sin que Sancho estuviera a su lado? Quizás esta pareja novelística sea uno de los ejemplos más evidentes del estar con. Cada uno de ellos se descubre en el espejo del otro y a través de esa relación se van transformando en un nosotros que mantiene las dos singularidades en su carácter finito. La ironía de Cervantes llega directamente de esa consciencia de finitud y es la que hace que la relación sea posible, sin que una subjetividad fagocite a la otra.

Precisamente es en el Quijote de Cervantes cuando la novela empieza a tener conciencia de sí misma y se establece como una forma peculiar de mirar la realidad. Siguiendo el pensamiento de Nancy en La comunidad inoperante, la novela sería un conocimiento experiencial del mundo como manera de existir juntos. "Un mundo no es ni espacio ni tiempo: es nuestra manera de existir juntos. Es nuestro mundo, el mundo de nosotros, no como una posesión sino como la apropiación de la existencia en cuanto finita, en cuanto que su propia esencia (existir) es al mismo tiempo llegar a un mundo y abrirlo. Pero este tiempo no es el de un origen, ni el origen del tiempo: existe "al espaciar el tiempo del tiempo, al abrir la posibilidad de decir «nosotros» y de enunciar y de anunciar por este «nosotros» la historicidad de la existencia. La historia no es un relato, ni un informe; es el anuncio de un «nosotros» (y en este sentido es escritura)"5.

El impulso novelístico al que estamos haciendo referencia surge de manera transversal a la historia, y los textos que nacen de ese impulso buscan esa experiencia de comunidad a través de una crítica especular de su presente. Podemos considerar la novela como la presencia de cada momento histórico al hilo de ese anuncio de un "nosotros". De manera que lo que nos muestra, lo que nos hace presente es cada nuevo mundo, "una época que, en suma, no puede pensarse más que como límite de época, si una «época» es una forma o un aspecto del «mundo»"6.

La importancia de la presencia de esos mundos que se van abriendo radica en el punto de vista del que parte, el de espejo crítico al margen de la ideología, con la consecuente necesidad de hacer presente, mostrar y no decir. Y esta intención de presencia, que ya despunta en la época helenística, se ha ido desarrollando a lo largo de la historia con distintas técnicas según el tipo de realidad que necesitara mostrar ${ }^{7}$. Se

\footnotetext{
${ }^{4}$ Sobre este tema, Nicolaï Hartmann desarrolla la idea de "dramatismo de la vida" como el aparecer del hombre en su convivencia con otros, en su encontrarse y chocar. Resultan interesantes algunos puntos en común entre el pensamiento de Nicolaï Hartmann y Jean-Luc Nancy que se refieren justamente al aparecer, la singularidad y la intersubjetividad. Ver N. Hartmann, Estética, México, Universidad Nacional Autónoma de México, 1977.

${ }^{5}$ J. L. Nancy, op. cit., p. 126.

${ }^{6}$ Ibidem, p. 103.

${ }^{7}$ Wayne C. Booth revolucionó la crítica narrativa con su distinción entre mostrar y decir, señalando el mostrar como un elemento fundamental en los textos novelísticos. Véase W.C. Booth, The rethoric of fiction, Chicago, University of Chicago Press, 1983.
} 
puede decir que la novela, abierta técnicamente, se ha encaminado hacia una presencia cada vez más potente que pone el foco en distintos ámbitos vitales dependiendo de cada nueva relación abierta entre individuo y sociedad y su dimensión deficiente con respecto al desarrollo del individuo como ser singular.

En su objetivo de criticar la evolución social desde una búsqueda de las lógicas internas de la condición humana, la presencia se ha ido conformando como fundamental. Mientras la evolución social ${ }^{8}$ camina hacia un individuo cada vez más alejado de la experiencia, hacia formas de organización al dictado de ideologías y la creación de masas, la novela avanza en mostrar con más intensidad la necesidad de experiencia para el desarrollo de cada uno de los miembros organizados.

En todos estos textos que tienen en común esa búsqueda se manifiesta la necesidad de experiencia, y esa necesidad que mueve la narración culmina en la experiencia de comunidad que constituye la propia novela, a la que el lector accede como una necesidad colmada.

La técnica ${ }^{9}$, fundamental en la novela, abierta y necesariamente original para poder mostrar la concreta forma de organización social de cada época creando una experiencia diferenciada, evoluciona en su manera de crear presencia. Es necesario hacer un pequeño recorrido por algunas novelas que ilustran ese proceso histórico del foco de la presencia.

La historia de Dafnis y Cloe ${ }^{10}$, contada por Longo en el siglo II, todavía es una historia cerrada por la fuerza del mito, el destino. Los personajes se cumplen a sí mismos asistidos por los dioses. Ellos se viven decidiendo, dudando, buscando, intentando cumplir su conatus $^{11}$, pero sin la ayuda de los dioses no podrían hacerlo. Y es esa consciencia de sí mismos, esa intuición de su conatus, lo que muestra Longo de

\footnotetext{
${ }^{8}$ La evolución social entendida desde el concepto de Gesellschaft. Nancy define la sociedad como "la asociación disociante de las fuerzas, de las necesidades y de los signos". Ver J. L. Nancy, La comunidad inoperante, Santiago de Chile, LOM, 2000, p. 23.

${ }^{9}$ M. M. Bajtín ha tratado la necesidad de definir la novela y la dificultad de hacerlo precisamente por estar en continuo movimiento y reflejar los cambios de la realidad. Esos cambios exigen técnicas diferentes, aunque todas ellas asumen la incorporación del presente a la narración. Ver el capítulo de La estilística contemporánea y la novela en Mijaíl M. Bajtín, Teoría y estética de la novela, Madrid, Taurus, 1989, pp. 78-93.

${ }^{10}$ Longo, Dafnis y Cloe, Barcelona, Libros Río Nuevo, 1990.

${ }^{11}$ La filosofía de Spinoza resulta fundamental en el análisis de los personajes de la novela en general. Todos los protagonistas transparentan un conatus, una inclinación que responde a su singularidad y que además tiene que ver con su pertenencia a la naturaleza, pertenencia siempre anulada por la organización social. Ese conatus siempre actúa como elemento detonante del conflicto que da lugar a la construcción de la novela. Este tema ha sido trabajado por Jordi Claramonte en los guiones del western. Ver J. Claramonte, Desacoplados. Estética y política del Western, Madrid, Ed. Papel de Fumar, 2010.
} 
una manera vívida, a través de la experiencia. ¿Cómo podemos ver el conflicto entre su singularidad y una sociedad que les obliga a ser lo que tienen que ser desde su esquema organizativo? Sintiéndolo. Esa vivencia la construye Longo haciendo aparecer el cielo, el mar, el olor de las flores, la calidez de la piel o la humedad del beso que experimentan los amantes en contraste con el comportamiento no natural de los ciudadanos, dueños de las tierras, que llegan al campo a hacer cumplir el orden. La presencia en este momento se resuelve en sensaciones. El simple hecho de asistir de forma sensitiva a la Grecia del siglo II, de tener delante sus modos de relación y el impacto que ejercen sobre sus habitantes, genera un conocimiento específico, hasta entonces inexistente en la literatura.

Esta intención narrativa nueva, de búsqueda, no unívoca, que se aleja del mito, se mantiene en Japón en el siglo X con La historia de Genji ${ }^{12}$ de Murasaki Shikibu, un texto donde el protagonista está en conflicto con su cerrado mundo de palacio. Su empeño por tener vivencias, por sentirse mientras conoce algo fuera de él, es la raíz de muchas historias que van conformando su personalidad y que dejan ver al lector una organización social limitante. Sin embargo, no se mantiene en Occidente, donde las narraciones se aferran a la representación de un ideal. Lejos de ser obras sensitivas, su pretensión es contar los valores fundamentales, la obediencia al ideal. El protagonista sigue una intuición que se acopla al ideal. En El caballero de la carreta ${ }^{13}$ de Chrétien de Troyes, Lanzarote sigue ciegamente lo que Amor le pide, escucha a Razón cuando tiene que ser cauto, pero Razón y Amor no responden a una manera singular de ver el mundo, sino a unas leyes ideales que todo caballero jura fielmente seguir.

Por eso, aunque resurge ese espíritu de crear experiencia ya en textos como El Lazarillo de Tormes, es el Quijote de Cervantes el que plantea de manera abierta algunas lógicas de la condición humana que van a aparecer en todas las novelas: el abandono de la ideología, la necesidad de la experiencia, la importancia de seguir el propio conatus, la relación como necesaria para conquistar una identidad. El planteamiento de Cervantes es abierto porque construye una novela dialéctica con dos mundos en diálogo marcados por la presencia. Seguimos la línea sensitiva de Longo, pero se ha incorporado el diálogo como técnica fundamental de presencia. Los personajes están ahí, hablando, transparentando su forma de pensar y el mundo que les ha llevado a conformar su pensamiento. Por un lado, Don Quijote, acoplado al ideal del caballero y por otro Sancho, desconocedor de ese ideal, en contacto con la naturaleza y la experiencia. Los dos se transforman en esa relación, cada uno de ellos va conformando una identidad hasta llegar a liberarse de la enajenación. Dice Don Quijote al final: "Yo fui loco y ya soy cuerdo: fui Don Quijote de la Mancha, y yo soy agora,

\footnotetext{
${ }^{12}$ Murasaki Shikibu, La historia de Genji, Mas Pou, Atalanta, 2006.

${ }^{13}$ Chrétien de Troyes, El caballero de la carreta, Madrid, Alianza Editorial, 1996.
} 
como he dicho, Alonso Quijano el Bueno. Pueda con vuestras mercedes mi arrepentimiento y mi verdad volverme a la estimación que de mí se tenía, y prosiga adelante el señor escribano"14.

En ese viaje dialéctico el lector siente la necesidad de liberarse de los cánones, de empezar a ver, a entender el mundo como relación. Aparece la posibilidad de relacionarse con el paisaje, con el compañero de viaje, una posibilidad de relación que está sujeta a liberarse de la condición, del ser representativo, incluso Sancho, también representante del mundo campesino, tiene que liberarse de sus límites sociales. El diálogo, que hasta entonces era meramente informativo, aparece como una nueva técnica novelística encaminada a crear experiencia, a dar un paso más en su evolución como presencia.

A partir de entonces, ese abandono del juego de la representación hacia la conciencia del individuo como proyecto acrecienta el impetus ${ }^{15}$ de la novela. Se va haciendo consciente de sí misma, de su campo de acción, de su impulso como búsqueda desde la crítica de lo dado. El descubrimiento del individuo se afianza en un descubrimiento de la singularidad como único camino que garantiza la relación.

Cervantes se había empeñado en terminar con la representación del ideal cuando surge Goethe impulsando la creencia en un mundo nuevo con el nacimiento de la burguesía, todavía una organización social en proceso de definición. En Los años de aprendizaje de Wilhelm Meister ${ }^{16}$ nos pone delante un individuo en formación, un individuo empeñado en conocer su singularidad, en seguir su conatus, que sólo puede alcanzar aprobando la singularidad de los otros. Cada uno de los miembros de esa sociedad ideal, sin ideología, basada en la posibilidad de desarrollo de sus miembros, tiene que descubrir su verdad por sí mismo. Es la experiencia la que proporciona esa sabiduría que hace que alguien sea capaz de encarnar la singularidad y crear comunidad en sus acciones. La presencia se consolida en la experiencia de la naturaleza, los diálogos y ya, además, las emociones explícitas. El protagonista no obedece a Amor, dialoga con él y lo descubre en la experiencia. El Wilhelm Meister da cuenta

\footnotetext{
${ }^{14}$ Miguel de Cervantes, Don Quijote de la Mancha, Madrid, Lunwerg, 2011, p. 1096. Este pasaje también está tratado desde la sensibilidad con un cambio radical del tempo de la novela. La llegada al fin de viaje, la asunción de la propia identidad, la posibilidad de tomar decisiones y formar parte del entorno, de la red comunitaria, se presenta mediante un efecto expresivo de sosiego, una compresión del tiempo y el espacio.

${ }^{15}$ En La partición de las artes, Jean Luc Nancy utiliza este término aplicado a la Filosofía y en ese mismo sentido se puede utilizar para describir el origen del novelar. Dice Nancy: "Filosofar no se da sin impulso, incluso sin un impulso violento que tire hacia delante y que desarraigue también: que desarraigue del sentido depositado, sedimentado, medio descompuesto; y que impulse hacia el sentido posible, sobre todo no dado, no disponible, que es preciso acechar, sorprender en su venida imprevisible y nunca simple, jamás unívoca". J. L. Nancy, op. cit., pp. 52-53.

${ }^{16} \mathrm{~J}$. W. von Goethe, Los años de aprendizaje de Wilhelm Meister, Madrid, Cátedra, 2008.
} 
del nacimiento de la autonomía simplemente como posibilidad de cumplimiento del propio proyecto y avanza en la presencia de las emociones.

Siguiendo este recorrido histórico de la novela y adelantándonos al siglo XIX, en concreto a la Revolución de 1848 en Francia presentada por Flaubert en La Educación Sentimental, la experiencia del lector, la posibilidad de vivir un momento ya pasado, terminado en el tiempo, se vuelve mucho más efectiva. La técnica novelística utiliza la presentación de hechos para crear un efecto, no para relatar, no para hacer un inventario o informar. Para sentir algo hay que utilizar los sentidos y esa presentación de elementos narrativos está creada para hacer sentir. La presencia se hace más sensitiva que nunca. Las interpretaciones históricas nunca pueden mostrar lo que allí ocurrió de una manera abierta, libre de interpretación. Como se muestra en el siguiente fragmento, los hechos nos hacen ver: "El palacio rebosaba gente. En el patio interior ardían siete hogueras. Se tiraban por las ventanas pianos, cómodas y relojes. Las bombas de incendio escupían el agua hasta los tejados. Unos hampones trataban de cortar las mangas con sus sables. Fréderic incitó a un alumno de la Escuela Politécnica a interponerse. El politécnico no comprendió, parecía imbécil. Alrededor, en las dos galerías, el populacho, dueño de las bodegas, se entregaba a una horrible borrachera. El vino corría a torrentes, les mojaba los pies, y los bribones bebían en botellas rotas, vociferaban y andaban titubeantes.

-"Salgamos de aquí -dijo Hussonet-, este pueblo me asquea"17.

En Ideas sobre la novela, Ortega y Gasset reflexiona sobre la evolución de la novela que "se ha ido desplazando de la pura narración, que era sólo alusiva, a la rigurosa presentación" 18 . Pone como ejemplo de escritura fallida la alusión a características de personajes que no nos ayudan a visualizarlos. "Nada de referirnos lo que un personaje es: hace falta que lo veamos con nuestros propios ojos" 19 .

Flaubert mostraba el aburrimiento como una primera señal de la imposibilidad del individuo de vincularse a su mundo. El siglo XX presenta un individuo que pertenece a una sociedad mucho más enajenante. La comunicación resulta imposible, al individuo en su conatus, en su deseo de ser singular, ya sólo le queda sentir encerrado en un mundo emocional no compartido. La presencia se amplía con la presencia del interior del personaje. El lector asiste sin mediación del narrador a su flujo de conciencia, a las emociones que fundan su lenguaje, un lenguaje que no comunica, que sólo puede comprender el lector. El individuo vive una especie de lucha a muerte por sobrevivir como singularidad consciente de no tener posibilidades para ello. La sociedad se ha convertido en una masa de individuos que obliga a la igualdad desde la nada. El personaje sólo se reconoce como singular en su emoción contra esa situación.

\footnotetext{
${ }^{17}$ Gustave Flaubert, La educación sentimental, Madrid, Alianza Editorial, 2011, p. 421.

${ }^{18}$ José Ortega y Gasset, Meditaciones del Quijote, Madrid, El arquero, 1975, p. 157.

${ }^{19}$ Ibidem, p. 157.
} 
La nueva técnica, el expresionismo, cambia el estilo de la novela pero sigue creando presencia, la expresión es presencia emotiva. Dentro de este expresionismo, de la presencia de la conciencia, la lista de autores es larguísima, autores de todos los países que coinciden en la necesidad de mostrar precisamente eso: Proust, Joyce, Woolf o Faulkner. En ese mismo momento, otros prefieren contrastar ese mundo interior con la sociedad vigente, marcando también la presencia de una forma de organización muerta, fuera de juego. Es el caso de Musil o Thomas Mann.

Este recorrido por algunos momentos históricos de la novela, en el que se puede ver la transformación del estilo o de la forma de crear presencia en función de los cambios de sensibilidad, ha alumbrado la novela precisamente como una modalidad literaria que muestra nuestras formas de organizarnos desde el deseo, la voluntad, incluso la necesidad de un mundo comunitario, una forma de existir juntos como relación de seres singulares.

\section{La novela como experiencia de comunidad}

En el capítulo anterior se revelaba la presencia como un recurso común para poder reflejar el conflicto entre individuo y sociedad en textos constatados como novelas. Se percibía ese reflejo como derivado de un espíritu crítico, un malestar en relación a la forma de organización social vigente. La evolución de la presencia se relacionaba con la especificidad de las maneras de imposibilitar el desarrollo del individuo como ser singular y por lo tanto, de imposibilitar la relación entre individuos. De manera que la crítica social también da identidad a la novela, una crítica en absoluto discursiva que responde simplemente a la necesidad de ver el escenario en el que vivimos, que por ser nuestro campo de acción, no resulta visible, como describe Nancy en La partición de las artes: "Estamos en un mundo y no delante de él. De ese modo, podemos también decir que no vemos jamás un mundo: estamos en él, lo habitamos, lo exploramos, nos encontramos en él o en él nos perdemos..."20.

Esta crítica social no parte de ningún presupuesto ideológico, ni religioso, en general de ninguna concepción del mundo. El impulso del que hemos hablado en el capítulo anterior, la búsqueda de las lógicas de la condición humana, es el que evidencia los errores en nuestra forma de organizarnos. Es una búsqueda que no encuentra, pero que obliga a ver, a reconocer lo que no es adecuado. Y precisamente ese no encontrar es lo que convierte a la novela en una experiencia en sí misma, una experiencia vital, que como toda experiencia vital no es instrumento para llegar a otro punto.

La presencia no busca el realismo, ni copiar la realidad, surge de una necesidad de crear experiencia. La búsqueda se hace experiencia y la experiencia colma el impulso.

\footnotetext{
${ }^{20}$ J. L. Nancy, op. cit., p. 87.
} 
Se podría definir la novela como un conocimiento experiencial del mundo como manera de existir juntos, como ya hemos visto. En el origen de la novela está abandonar el ideal que lleva a la representación. En este sentido no crea comunidad como el mito, no nace de la comunidad del ser común, no pretende cerrar un mundo, un significado, sino que muestra lo contrario: la necesidad de apertura como condición de humanidad, la necesidad de singularidad y de relación como acto de comunidad.

La reflexión de Nancy sobre el mito interrumpido, el mito consciente de sí mismo como mito, por lo tanto sin capacidad de crear comunidad o la pregunta por la posibilidad de una comunidad nueva si no hay una mitología nueva, puede descubrir la novela como cierta sustitución del mito, una forma de crear estructura comunitaria basada en la experiencia de comunidad. Dice Nancy sobre la literatura mítica en La comunidad inoperante: "En el mito o en la literatura mítica, las existencias no están ofrecidas en su singularidad: sino que los trazos de particularidad contribuyen al sistema de «una vida ejemplar», de la cual nada se retira, donde nada permanece más acá de un límite singular, donde todo se comunica y se impone a la identificación"21.

La experiencia tiene en su base la singularidad. No es posible vivir una experiencia desde lo ajeno. Lo común como una abstracción no es susceptible de ser experimentado. La literatura de la experiencia, la novela, no busca la comunicación partiendo de supuestos comunes. En su forma de presentar la lucha por la singularidad está haciendo aparecer la comunidad. La comunidad aparece en el hecho de compartir la finitud, de sentir esa finitud compartida. La novela puede ser la forma literaria de la comunidad interrumpida, la comunidad sin mito, el arte que expone, que hace aparecer la comunidad. En realidad, el problema de la comunidad perdida podría ser visto al revés. ¿Ha habido una comunidad real con el mito? La comunidad es un acto, una experiencia que no se puede dar en un mundo de representación. Nancy alude a Bataille en La comunidad inoperante para resaltar su idea de que "no hay experiencia fuera de la comunidad" 22 . También alude a la comunicación como constituyente de la realidad humana. En este sentido, la novela, que busca las lógicas internas de la condición humana, encuentra una de ellas en sí misma como experiencia de comunicación, experiencia que se repite en todos y cada uno de los textos y colma el impulso originario.

En la novela es la singularidad lo que está en jaque. Su crítica se dirige siempre a las formas de organizarnos que niegan la posibilidad de serlo, de ser en común, de

\footnotetext{
${ }^{21}$ J. L. Nancy, op. cit., p. 93. Este capítulo de "El comunismo literario" interesa a este artículo en cuanto a la aparición del ser singular como diferente del individuo y del ser común. La novela siempre construye personajes en busca de espacio para desarrollarse en su singularidad. La vida ejemplar supone exactamente lo contrario, el cumplimiento como individuo, sólo como parte de un todo ajeno.

${ }^{22}$ J. L. Nancy, op. cit., p. 32.
} 
vivir en relación, de tener existencia en el sentido de ser reconocido y reconocerse. Critica cualquier intento de encerrar al hombre en un concepto o crear un mundo cerrado en un ideal. A lo largo de la historia, evidencia unas veces un exceso de individualismo y otras un exceso de colectivismo. De manera que la novela narra desde un impulso o deseo de comunidad que aparece como una de las lógicas de la condición humana. El campo de la novela es el de la comunidad: en la crítica a una forma de organizarnos que anula la singularidad y la posibilidad de relación, construye una experiencia de comunidad. Desde esa experiencia de comunidad adoptamos la distancia necesaria para ver nuestro mundo. Lo que no entendemos es la finitud y lo que narramos son nuestras formas erróneas de enfrentarnos a ella. En este sentido, la novela es un conocimiento experiencial del mundo como forma de estar juntos, de las distintas formas de estar juntos, reconstruyendo esos mundos en presencia desde el punto de vista de la experiencia de comunidad. La novela repite una y otra vez de distintas maneras que la finitud tiene que ver con la búsqueda y no con el hallazgo. Leemos novelas para estar ahí, para encontrarnos en comunidad, sintiéndonos parte del mundo, en el sentido de reparto ${ }^{23}$ al que se refiere Nancy. Encontrarnos en comunidad puede ser pensado como una vivencia del nosotros de Nancy que describe en Ser singular plural: “«Nosotros» no dice ni el «Uno», ni la suma de los «unos» y de los «otros», sino que «nosotros» dice «uno» de una manera singular plural, uno por uno y uno con uno" 24 .

Retomando el inicio de este artículo tenemos que preguntarnos: ¿Sigue siendo la novela en nuestros días una forma de arte adecuada a nuestro momento vital, a nuestra sensibilidad actual? El movimiento literario llamado postmoderno ${ }^{25}$ parece que ha ocupado el espacio literario como la nueva forma de narrar que refleja nuestro mundo. Muchos de los escritores que siguen esta tendencia consideran imposible escribir una novela en estos momentos. Si se mantiene el recorrido histórico de la evolución en las relaciones entre individuo y sociedad, la narrativa postmoderna estaría contando el momento en el que el individuo se ha desvinculado definitivamente de su mundo. La búsqueda se habría terminado y la posibilidad de experiencia con ella. Con el individuo encapsulado y olvidado de su posibilidad de ser singular, el conflicto

\footnotetext{
${ }^{23}$ Resulta muy interesante cómo Nancy en La comunidad inoperante asemeja el reparto del límite con "ese enlace donde el mito mantiene juntos y estructura a los hombres, a los dioses, a los animales y a la totalidad del mundo". J. L. Nancy, op. cit., p. 92.

${ }^{24}$ J. L. Nancy, op. cit., p. 92.

${ }^{25}$ Con narración postmoderna nos referimos a una forma de narrar con origen en la novela, pero desligada de ella. La distinción que aquí se pretende es de forma, pero atendiendo al impulso, al carácter abierto, de búsqueda, a la no representación de una idea propia de la novela. Postmoderno no significa más que después de lo moderno, en ese sentido la novela también puede ser postmoderna, ya que el objetivo de este artículo es hacerla ver como transversal a la historia.
} 
no existe. Desde esa intención narrativa, la presencia deja de tener sentido, no se quiere ver lo que no podemos ver en nuestra vida cotidiana, se quiere contar lo que ya sabemos que ocurre. La narración vuelve a ser una representación, la representación de un estilo de vida que otros campos analizan ${ }^{26}$. Es descripción en el sentido de sucesión sin posibilidad de acontecimiento. Los personajes no se pueden encontrar, ni pueden revelar nada en común con el lector porque no muestran el mundo que no pueden ver, sino que forman parte de la descripción de lo que se ve. El verdadero protagonista en esta forma de narrar es el lenguaje, que se cuenta a sí mismo, que implica la desaparición de los sentidos, el final del mostrar y la vuelta al decir, aunque de otra manera. La narración postmoderna, no abierta, representativa de un canon preestablecido no supone la muerte de la novela, sino otro camino narrativo de tipo descriptivo. Un camino narrativo que cuenta la absoluta fragmentariedad, la absoluta falta de relación del individuo con lo otro, la absoluta desaparición de la singularidad. Ese posicionamiento no puede responder al mundo como lugar de acción, sino que necesariamente responde a una idea de mundo, una abstracción que procede del hecho real de la falta de vínculo del individuo con su mundo, pero que abandona la búsqueda y la presentación abierta de los hechos y se convierte en un juego virtual.

Este camino narrativo, diferente de la novela al abandonar su impulso original, no significa la muerte efectiva del conflicto, la búsqueda y la crítica. La narración postmoderna nace precisamente de la evolución de la novela, pero se desprende de ella. Eso no anula la experiencia de comunidad de la novela como respuesta a una necesidad actual de salida del yo y encuentro con lo otro como forma de reconocernos en nuestra finitud compartida. El recorrido de la novela puede dar formas narrativas que se desligan de su impulso original sin que la anulen. Quizás se pueda decir que la novela supuso un adelanto de tipo artístico, intuitivo, a las teorías filosóficas sobre la comunidad y que a partir de ellas tiene por delante un nuevo camino con más consciencia de sí misma como forma de arte autónoma.

\footnotetext{
${ }^{26}$ Es propio de la narrativa postmoderna adoptar formas de expresión de otros campos de conocimiento, como el científico y aplicarlos a la literatura. Aunque el primer libro postmoderno pudo adoptar estos modelos respondiendo a una necesidad narrativa, a partir de entonces, la técnica dejó de ser original y se convirtió en un modelo.
} 